\title{
Optimal truss and frame design from projected homogenization-based topology optimization
}

\author{
Larsen, S. D.; Sigmund, O.; Groen, J. P.
}

Published in:

Structural and Multidisciplinary Optimization

Link to article, DOI:

10.1007/s00158-018-1948-9

Publication date:

2018

Document Version

Peer reviewed version

Link back to DTU Orbit

Citation (APA):

Larsen, S. D., Sigmund, O., \& Groen, J. P. (2018). Optimal truss and frame design from projected

homogenization-based topology optimization. Structural and Multidisciplinary Optimization, 57(4), 1461-1474.

https://doi.org/10.1007/s00158-018-1948-9

\section{General rights}

Copyright and moral rights for the publications made accessible in the public portal are retained by the authors and/or other copyright owners and it is a condition of accessing publications that users recognise and abide by the legal requirements associated with these rights.

- Users may download and print one copy of any publication from the public portal for the purpose of private study or research.

- You may not further distribute the material or use it for any profit-making activity or commercial gain

- You may freely distribute the URL identifying the publication in the public portal

If you believe that this document breaches copyright please contact us providing details, and we will remove access to the work immediately and investigate your claim 


\title{
Optimal truss and frame design from projected homogenization-based topology optimization
}

\author{
S. D. Larsen · O. Sigmund · J. P. Groen
}

Received: date / Accepted: date

\begin{abstract}
In this article, we propose a novel method to obtain a near-optimal frame structure, based on the solution of a homogenization-based topology optimization model. The presented approach exploits the equivalence between Michell's problem of least-weight trusses and a compliance minimization problem using optimal rank2 laminates in the low volume fraction limit. In a fully automated procedure, a discrete structure is extracted from the homogenization-based continuum model. This near-optimal structure is post-optimized as a frame, where the bending stiffness is continuously decreased, to allow for a final design that resembles a truss structure. Numerical experiments show excellent behavior of the method, where the final designs are close to analytical optima, and obtained in less than 10 minutes, for various levels of detail, on a standard PC.
\end{abstract}

Keywords Optimal frame design · Optimal truss design $\cdot$ Michell theory $\cdot$ Topology optimization

\section{Introduction}

A classical topic within structural optimization is to find solutions for Michell's problem of least-weight trusses (Michell, 1904). Computational methods to solve these problems of optimal truss design date back to the early sixties, when Dorn et al. (1964) introduced the ground structure approach. This approach requires a fixed set of nodal joints and elements, which make up the ground structure. The cross-sectional areas of

S. D. Larsen · O. Sigmund · J. P. Groen

Department of Mechanical Engineering, Solid Mechanics, Technical University of Denmark, 2800 Kgs, Lyngby, Denmark

Tel.: +45-45254252

E-mail: jergro@mek.dtu.dk these elements are then optimized, classically using linear programming methods. In recent years two easy-touse implementations have been presented. Sokól (2011) has published a 99 line code programmed in Mathematica, while Zegard and Paulino (2014) present a frame work for arbitrary 2D domains in MATLAB.

A downside of ground structure approaches is that the location of the nodal joints has a large influence on the performance of the design. To get a near-optimal solution a large set of nodes and potential elements have to be considered. Furthermore, the large number of members and the fact that some of these members are overlapping poses a limit on the manufacturability of these designs.

It is also possible to include the location of the nodes as design variables, i.e. both size and geometry are optimized, as introduced by Dobbs and Felton (1969) and Pedersen (1969). In this case a small set of nodes and elements suffices to get a near-optimal design; however, due to the non-linearity of the combined size and geometry optimization problem the initial position of nodes and connectivity still has a large influence on the result.

To get close to the optimal distribution of nodes and elements, growth methods have been considered (Rule, 1994). Martínez et al. (2007) introduce an efficient growth method where sequentially a node and elements are introduced, size and topology optimization are performed, and geometry optimization is applied. The resulting designs are close in performance to analytical solutions for Michell's problem of least-weight trusses and obtained in a relatively short time. However, due to the nature of the heuristics involved in the growth method, it may not always converge to a near-optimal solution as is discussed by He and Gilbert (2015). Another downside of the growth method is that the procedure of finding an appropriate position to in- 
sert a new joint becomes increasingly slow when more members are considered.

In an approach somewhat related to the present, He and Gilbert (2015) make use of an efficient ground structure method, in which not all members are considered initially, but adaptively inserted (Gilbert and Tyas, 2003). The position of this set of nodes is then optimized in a subsequent geometry optimization step. Furthermore, crossing elements are treated by inserting new nodes at crossings, leading to near-optimal and manufacturable designs in a short time.

In a different approach Zhou and Li (2008, 2011) use truss-like continua to get a near-optimal distribution of material and orientations. In a semi-automated approach starting points for ray tracing are manually selected, the grid formed by these rays is interpreted as a truss structure, on which subsequent size and geometry optimization is performed. Similar to this approach, Gao et al. (2017) obtain an initial ground structure for size optimization, using principal stress trajectories. These trajectories are obtained when the domain is modeled as an isotropic medium; however, here it should be mentioned that these principal stress lines do not necessarily correspond to the principal directions for an orthotropic truss-like material. In both of the above mentioned approaches the initial member areas are not chosen based on the continuum model, but are found in a subsequent sizing optimization model.

In this article, we propose a novel method to obtain a near-optimal set of nodes and elements, based on the solution of a homogenization-based topology optimization model. The approach is fully automatic and the extracted structure remains close to optimal. In a final step, the nodal positions and element areas are further optimized, and redundant nodes and elements eliminated. An overview of the proposed method can be seen in Figure 1.

As discussed by Bendsøe and Haber (1993), the compliance minimization problem in the low volume fraction limit, using optimal rank-2 laminates, reduces to Michell's problem of least-weight trusses. In this limit a rank-2 laminate can be represented by a combination of two orthogonal rank-1 laminates acting on the same length-scale (Bourdin and Kohn, 2008), similar to the truss-like continua used by Zhou and Li (2008). Such a microstructure can be projected on a fine scale using the method proposed in Pantz and Trabelsi (2008) and Groen and Sigmund (2017). However, we can also use the information of the mapping functions, required to do the projection, to establish nodes and initial connectivity. Furthermore, based on the optimal material distribution of the continuum model, a near-optimal initial starting guess for element areas is obtained.
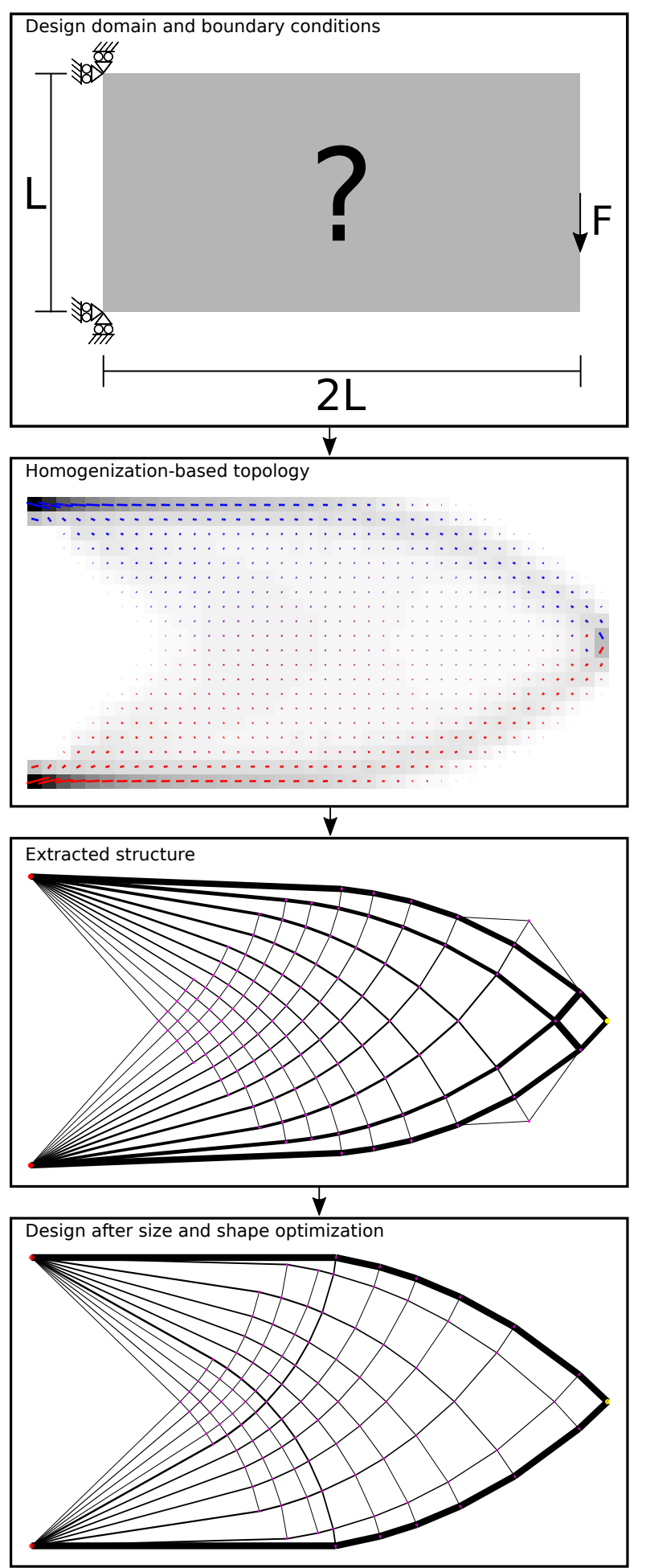

Fig. 1: Proposed procedure to obtain a solution to Michell's problem of least-weight trusses for a Michell cantilever

Although the projected structure is close to the optimal solution, it is not always in the space of statically determinate structures. Hence, when modeled as a truss 
structure, the stiffness matrix can be singular. To tackle the problem of a singular stiffness matrix in the context of topology optimization several approaches have been proposed. Bruns (2006) uses a pseudo-inverse method, Washizawa et al. (2004) use Krylov subspace methods, and Ramos Jr. and Paulino (2016) use a potential energy approach with Tikhonov regulation. However, as a simple and reliable alternative we choose to model the structure as a thin frame structure and gradually decrease the bending contribution using a continuation scheme. As an added benefit, our approach allows to study the relation between optimized frame and truss structures.

The combined procedure of obtaining a nearoptimal initial structure and post-optimization, for designs with several hundreds of nodes, requires less than 10 minutes, using a single processor MATLAB code on a standard PC. This short time potentially allows designers to use topology optimization as an interactive tool in the design process, (Aage et al., 2013). To demonstrate the performance of the proposed approach five examples are considered. The cantilever beam shown in Figure 1, and the MBB-beam, MBB-beam with void domain, L-shaped domain and Michell cantilever with circular support shown in Figure 2(a-d), respectively. The paper is organized as follows: The methodology of the homogenization-based topology optimization is introduced in Section 2. In Section 3 the theory used to obtain a near-optimal frame structure from projection of the rank-2 laminates is explained. The procedure to do further optimization is introduced in Section 4. The corresponding tests on the performance and efficiency of the developed method are shown in Section 5. Finally, the most important conclusions of this study are summarized in Section 6 .

\section{Homogenization-based topology optimization}

Two orthogonal rank-1 laminates are used as microstructure to perform the homogenization-based topology optimization. The microstructures are defined by $\mu_{1}$, and $\mu_{2}$, which are the relative widths of the isotropic material in layer 1 and 2 respectively, and angle $\theta$, which describes the angle between the material frame of reference to the global frame of reference. The corresponding constitutive properties $\mathbf{C}$ in the global coordinate system are written in matrix form using Voigt notation; furthermore, the macroscopic volume fraction $m$ can be calculated as,

$$
\begin{gathered}
\mathbf{C}\left(\mu_{1}, \mu_{2}, \theta\right)=\mathbf{R}^{T}(\theta)\left[\begin{array}{ccc}
E \mu_{1} & 0 & 0 \\
0 & E \mu_{2} & 0 \\
0 & 0 & 0
\end{array}\right] \mathbf{R}(\theta) \\
+\frac{E_{\min }}{1-\nu^{2}}\left[\begin{array}{ccc}
1 & \nu & 0 \\
\nu & 1 & 0 \\
0 & 0 & \frac{1-\nu}{2}
\end{array}\right]
\end{gathered}
$$

$m\left(\mu_{1}, \mu_{2}\right)=\mu_{1}+\mu_{2}$

Here $E$ is the Young's modulus of the isotropic material, and $\mathbf{R}$ is the transformation matrix rotating constitutive properties from the material frame of reference to the global frame of reference. For stability reasons a small isotropic stiffness $\left(E_{\min }=0.005 E\right.$ and $\left.\nu=0.3\right)$ is added to the composite constitutive properties.

Design domain $\Omega$ is discretized in $n_{e}$ bi-linear finite elements, each consisting of a uniform microstructure described by local design variables $\mu_{1}, \mu_{2}$, and $\theta$. The optimization problem, aimed at minimizing compliance $\mathcal{J}$, is solved in a nested-approach, where the design vectors describing the relative widths $\boldsymbol{\mu}_{1}$, and $\boldsymbol{\mu}_{2}$ are updated using the Method of Moving Asymptotes (MMA), (Svanberg, 1987). As discussed by Pedersen (1989, 1990), the optimal orientation of an orthotropic composite is along the directions of principal stresses, hence at each design iteration the angles $\boldsymbol{\theta}$ are updated accordingly. In Michell's problem of least-weight trusses the relative material distribution has to be found, hence $\boldsymbol{\mu}_{1}$ and $\boldsymbol{\mu}_{2}$ are only bounded from below, and the volume constraint can be arbitrarily set to one. The discretized optimization problem can thus be written as,

$$
\begin{aligned}
\min _{\boldsymbol{\mu}_{1}, \boldsymbol{\mu}_{2}, \boldsymbol{\theta}}: & \mathcal{J}\left(\boldsymbol{\mu}_{1}, \boldsymbol{\mu}_{2}, \boldsymbol{\theta}, \mathbf{U}\right)=\mathbf{F}^{T} \mathbf{U} \\
\text { s.t. }: & \mathbf{K}\left(\boldsymbol{\mu}_{1}, \boldsymbol{\mu}_{2}, \boldsymbol{\theta}\right) \mathbf{U}=\mathbf{F} \\
& : \mathbf{v}^{T} \mathbf{m}\left(\boldsymbol{\mu}_{1}, \boldsymbol{\mu}_{2}\right)-1 \sum_{e=1}^{n_{e}} v_{e} \leq 0 \\
& : \mathbf{0} \leq \boldsymbol{\mu}_{1}, \boldsymbol{\mu}_{2}
\end{aligned}
$$

Where $\mathbf{v}$ is the vector containing the element volumes $v_{e}$. Stiffness matrix $\mathbf{K}$ is a function of $\boldsymbol{\mu}_{1}, \boldsymbol{\mu}_{2}$, and $\boldsymbol{\theta}, \mathbf{F}$ is the load vector, and $\mathbf{U}$ describes the displacement field. To avoid checkerboard patterns due to the use of bi-linear finite elements, a standard density filter with a filter radius of 1.5 element widths is applied on both fields $\mu_{1}$ and $\mu_{2}$ independently, (Bour$\operatorname{din}, 2001$; Bruns and Tortorelli, 2001). Furthermore, as is proposed in Groen and Sigmund (2017), small values of $\mu_{1}$ and $\mu_{2}$ need to be prevented to make a clear distinction between regions consisting of material and 

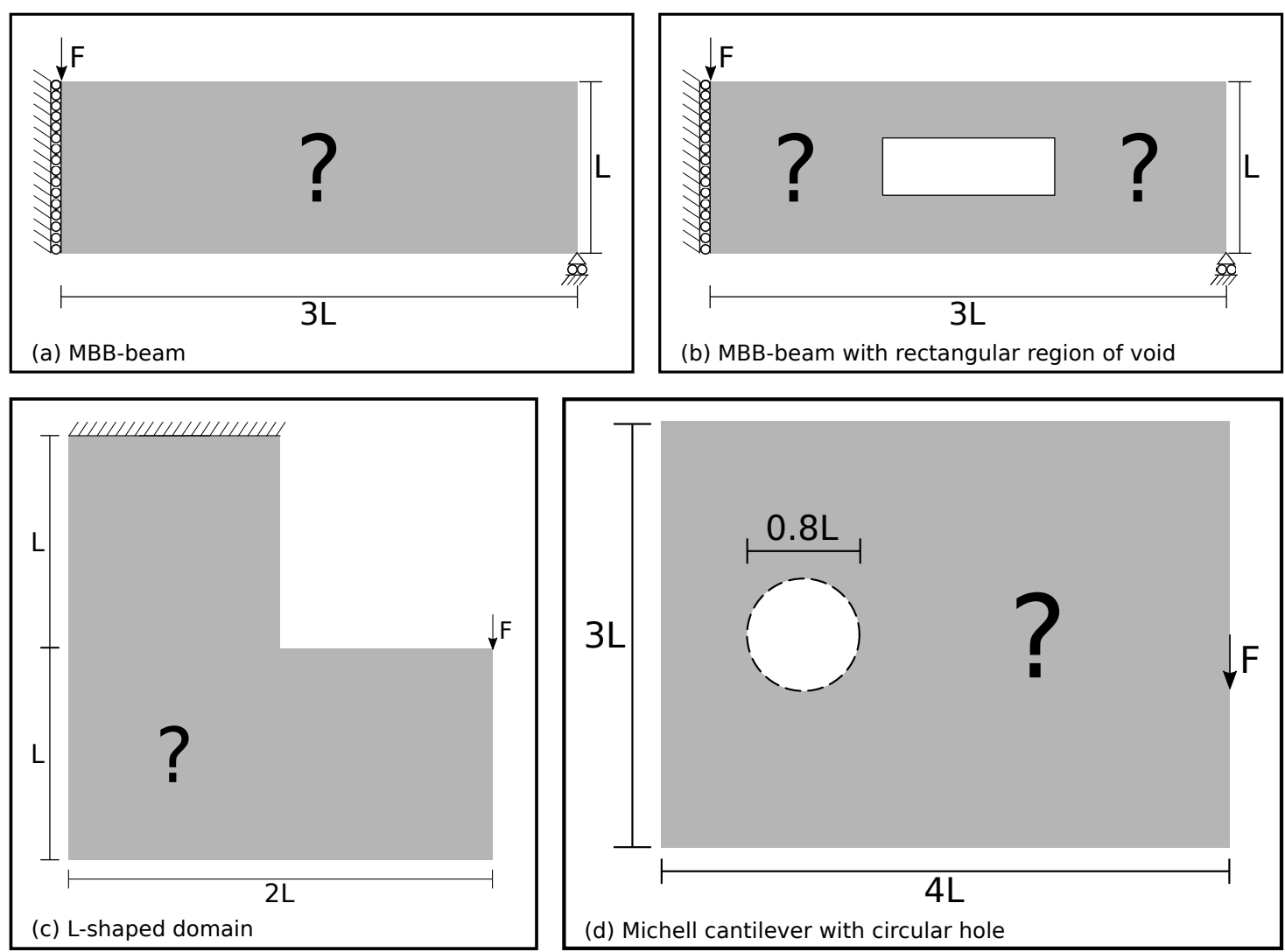

Fig. 2: Design domains and boundary conditions of examples used in this paper

regions which are void. To do so, the following material interpolation scheme is used,

$\overline{\tilde{\mu}}_{i}=\tilde{\mu}_{i} \frac{\tanh (\beta \eta)+\tanh \left(\beta\left(\tilde{\mu}_{i}-\eta\right)\right)}{\tanh (\beta \eta)+\tanh (\beta(1-\eta))}$

Where $\tilde{\mu}_{i}$ is the filtered width, and $\overline{\tilde{\mu}}_{i}$ is the physical laminate width used to calculate the constitutive properties. By carefully choosing a continuation scheme for the threshold parameter $\eta$, and the sharpness of the projection $\beta$, small widths between 0 and $\eta$ can be banned from the solution space with little effect on the performance of the design. The parameters used for the continuation approach can be found in Figure 3, where the legend shows the order of the scheme that is taken, using 50 iterations per step. Here the choice for $\eta=0.05$, means that no microstructures are allowed that contain less than $5 \%$ of the average volume.

\section{Mapping microstructures onto frame structure}

In recent works homogenization-based topologies have been projected as smooth and continuous lattice struc-

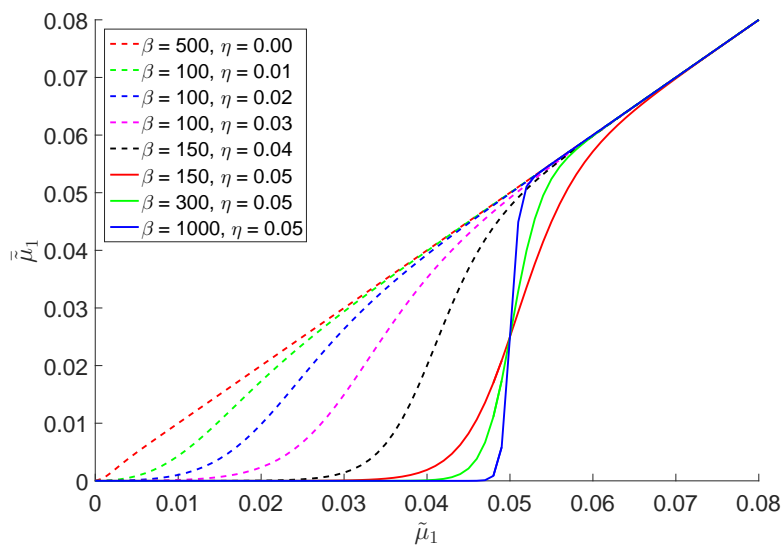

Fig. 3: Interpolation scheme plotted for different values of $\eta$ and $\beta$, the order of the lines follows the continuation approach

tures using two orthogonal layers.For each of the two orthogonal layers of the unit cell used in the homogenizationbased topology optimization, a mapping function is determined that is locally aligned with the direction of lamination. Using these two mapping functions, $\phi_{1}$ and 
$\phi_{2}$ respectively, a continuous sequence of unit cells can be projected by means of cosine functions.

In this section, we present an alternative method to create a near-optimal frame structure based on $\phi_{1}$ and $\phi_{2}$, which subsequently can be used for further optimization. The discussion on the derivation of the mapping functions will be kept limited, since this is not the main goal of this work. For a detailed derivation the reader is referred to Groen and Sigmund (2017).

\subsection{Mapping a periodic composite shape}

Mapping functions $\phi_{1}$ and $\phi_{2}$ can be obtained independently of each other, using a similar approach, therefore we restrict ourselves to the derivation of $\phi_{1}$. A suitable parameterization of $\phi_{1}$ has to fulfill two requirements:

1. $\phi_{1}$ should be constant in the direction of lamination in non-void domains, such that the frame structure can be described as contour lines.

2. The spacing between the contour lines of $\phi_{1}$ in these non-void domains, should be as regular as possible without violating the first requirement.

These requirements are not equally weighted throughout domain $\Omega$. As shown in Figure 1] a part of the homogenization-based topology does not consist of material, hence the mapping functions do not have to be accurate here. Furthermore, requirement 1 is too strict in regions where the angle field changes rapidly, e.g. at the Dirichlet BC's in the cantilever beam example. Therefore domain $\Omega$ is split in three subdomains, a smooth lattice domain $\Omega_{l}$, a void domain $\Omega_{v}$, and a domain $\Omega_{\theta}$ in which the angle field is rapidly changing. These domains are defined as,

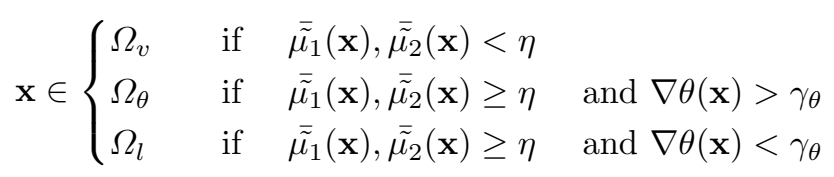

where, $\gamma_{\theta}$ is a threshold that dictates whether the angular field is rapidly changing or not.

$\gamma_{\theta}=\frac{\pi}{4} \frac{1}{h_{c}}$

Here $h_{c}$ is the element length used in the homogenizationbased topology optimization. Due to the selected threshold, $\Omega_{\theta}$ contains the parts of the domain where the angle field is close to singular. Using these different domains, we can solve for mapping function $\phi_{1}$ by means of a spatially weighted constrained least-squares minimization problem.

$$
\begin{aligned}
& \min _{\phi_{1}(\mathbf{x})}: \mathcal{I}\left(\phi_{1}(\mathbf{x})\right)=\frac{1}{2} \int_{\Omega} \alpha_{1}(\mathbf{x})\left\|\nabla \phi_{1}(\mathbf{x})-\mathbf{e}_{1}(\mathbf{x})\right\|^{2} \mathrm{~d} \Omega \\
& \text { s.t. }: \alpha_{2}(\mathbf{x}) \nabla \phi_{1}(\mathbf{x}) \cdot \mathbf{e}_{2}(\mathbf{x})=0
\end{aligned}
$$

where,

$$
\alpha_{1}(\mathbf{x})=\left\{\begin{array}{lll}
0.01 & \text { if } & \mathbf{x} \in \Omega_{v} \\
0.1 & \text { if } & \mathbf{x} \in \Omega_{\theta} \\
1 & \text { if } & \mathbf{x} \in \Omega_{l}
\end{array}, \quad \alpha_{2}(\mathbf{x})=\left\{\begin{array}{lll}
0 & \text { if } & \mathbf{x} \in \Omega_{v} \\
0 & \text { if } & \mathbf{x} \in \Omega_{\theta} \\
1 & \text { if } & \mathbf{x} \in \Omega_{l}
\end{array}\right.\right.
$$

Furthermore, unit vectors $\mathbf{e}_{1}, \mathbf{e}_{2}$ depend on the local directions of lamination $\theta$,

$\mathbf{e}_{1}=\left[\begin{array}{c}-\sin (\theta) \\ \cos (\theta)\end{array}\right], \quad \mathbf{e}_{2}=\left[\begin{array}{c}\cos (\theta) \\ \sin (\theta)\end{array}\right]$

It can be seen that the constraint, which dictates exact angular enforcement, is only active in $\Omega_{l}$, i.e. the part of the domain where the lattice is smooth. Experiments have shown that a high gradient in angle field in combination with angular enforcement results in a severely distorted lattice spacing, hence the calculation of $\phi_{1}$ in $\Omega_{\theta}$ is relaxed. Since $\phi_{1}$ does not have to be accurate in $\Omega_{v}$, its calculation is heavily relaxed to allow for the best approximation in other parts of $\Omega$.

Furthermore, it has to be noted that the principal stress directions used to calculate $\theta$ are rotationally symmetric, hence there may be jumps of size $\pi$ in angle field $\theta$. These jumps are identified using connected component labeling and aligned consistently as suggested in Groen and Sigmund (2017), to allow for a smooth projection using Equation 6 .

The mapping functions for the Michell cantilever are shown in Figure 4 (a). The microstructure is optimized on a coarse mesh of $80 \times 40$ elements. Afterwards, mapping functions $\phi_{1}$ and $\phi_{2}$ are calculated on a six times finer mesh of $480 \times 240$ elements, This is done to get smooth and accurate values for $\phi_{1}$ and $\phi_{2}$ yet still at low computational cost.

\subsection{Extraction of nodes and connectivity}

The contour lines of mapping functions $\phi_{1}$ and $\phi_{2}$, shown in Figure 4(b) resemble a frame-like structure. Using standard MATLAB functions (e.g. contour), these contour lines can be extracted, from which nodal positions and connectivity of the initial frame structure are established. To obtain highly accurate locations of the 


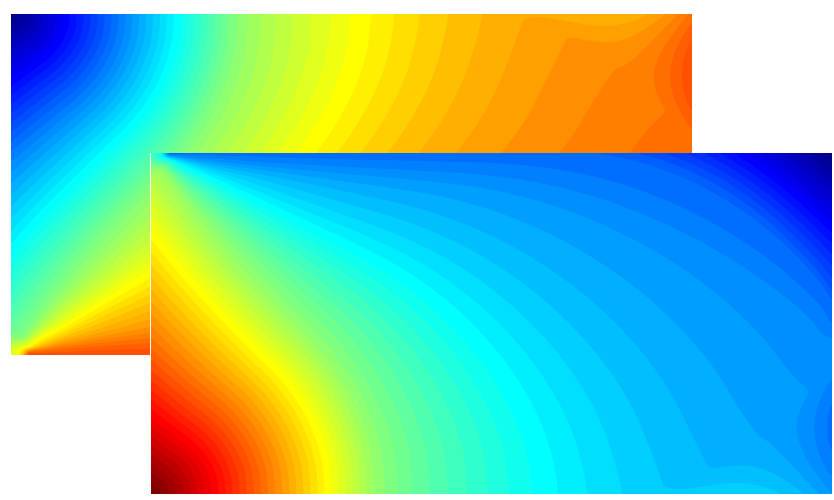

(a) Mapping functions $\phi_{1}$ and $\phi_{2}$

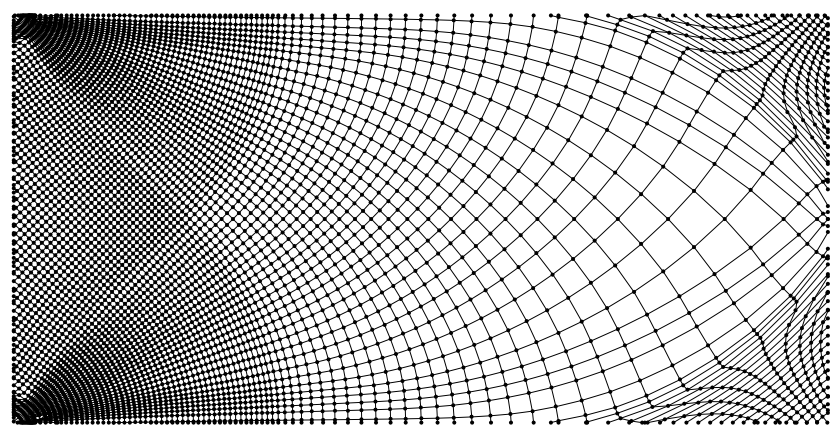

(c) Elements and nodes for $\varepsilon=20 h_{f}$

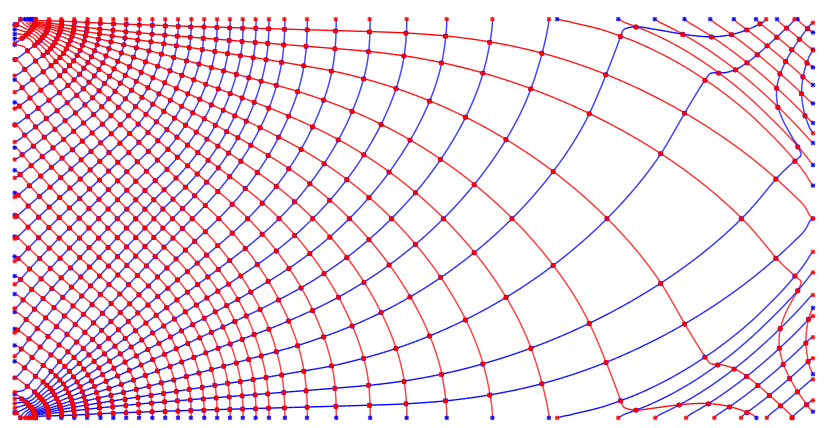

(b) Corresponding contour lines

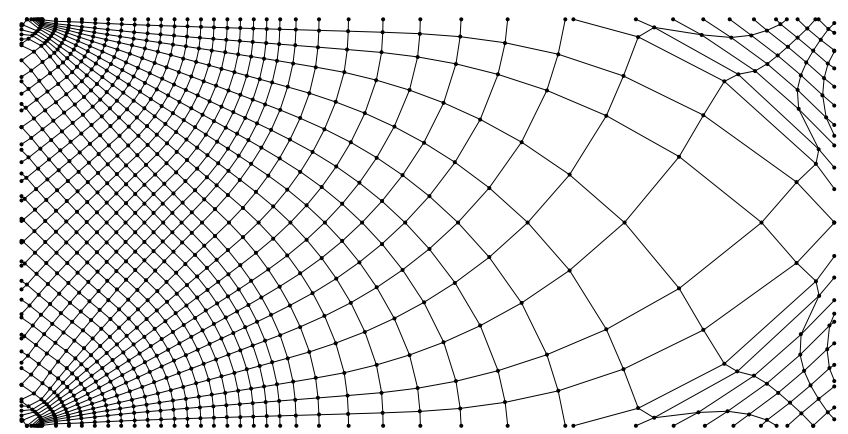

(d) Elements and nodes for $\varepsilon=50 h_{f}$

Fig. 4: The nodes and element-connectivity extracted from the mapping functions for the Michell cantilever problem

contour lines, mapping functions $\phi_{1}$ and $\phi_{2}$ are interpolated on a fine mesh of $1600 \times 800$ elements, i.e. 20 times finer than the mesh used for homogenizationbased topology optimization.

For simplicity, we choose to draw a contour line when mapping function $\phi_{i}$ takes a whole value. To influence the number of contour lines, $\phi_{i}$ is multiplied with periodicity scaling parameter $P_{i}$, which is based on a user-defined average length of a frame member $\varepsilon$.

$P_{i}=\frac{1}{\varepsilon} \frac{\int_{\left(\Omega \backslash \Omega_{v}\right)} \mathrm{d}\left(\Omega \backslash \Omega_{v}\right)}{\int_{\left(\Omega \backslash \Omega_{v}\right)}\left\|\nabla \phi_{i}(\mathbf{x})\right\|_{2} \mathrm{~d}\left(\Omega \backslash \Omega_{v}\right)}$

Where the integrals scale mapping functions $\phi_{i}$ w.r.t. their average spacing. It can easily be seen that a large value of $\varepsilon$ results in a small number of nodes and elements, while a small value results in a detailed frame structure. Finally, to make sure that a contour line passes through a specific point, e.g. the load-node, functions $\phi_{1}$ and $\phi_{2}$ can be shifted, before the contour-lines are extracted. An overview of the nodes and element connectivity for the Michell cantilever can be seen in Figure 4 here figures (c) and (d) show the nodes and connectivity for $\varepsilon=20 h_{f}$, and $\varepsilon=50 h_{f}$ respectively.

\subsection{Mapping of material distribution to element areas}

Relative areas are assigned to the frame elements based on the values of $\overline{\tilde{\mu}}_{1}$, and $\overline{\tilde{\mu}}_{2}$ from the homogenizationbased topology optimization. To transfer the continuous material distribution to the discrete elements, a polygon is drawn around each element, which describes the area in the continuum domain the element covers. An example of such a polygon can be seen in Figure 5(a). These polygons are obtained using the spacing of the contour lines, $w_{i}$, which can be approximated locally using mapping function $\phi_{i}$.

$w_{i}(\mathbf{x})=\frac{1}{\left\|\nabla \phi_{i}(\mathbf{x})\right\|_{2}}$

For each node of an element this spacing is calculated, and by taking a step orthogonal to the element with a stepsize of half the spacing the corresponding polygon can be drawn. By integrating the values of $\overline{\tilde{\mu}}_{1}$ or $\overline{\tilde{\mu}}_{2}$ in each of the polygons and dividing by the element length, a near-optimal initial area distribution of the frame structure is obtained based on the continuum solution, see e.g. Figure 5(b). Finally, we can identify the threshold area $A_{\eta}$, which is the area that an average sized frame element (i.e. with length $\varepsilon$ ) should have if 
$\overline{\tilde{\mu}}_{i}=\eta$

$A_{\eta}=\eta \varepsilon$

Bars that have an area smaller than this value, occur when a part of the polygon, used for integrating the volume, is within $\Omega_{v}$. Hence, elements with a mapped area that is smaller than $A_{\eta}$ are removed, as can be seen in Figure 5(c).

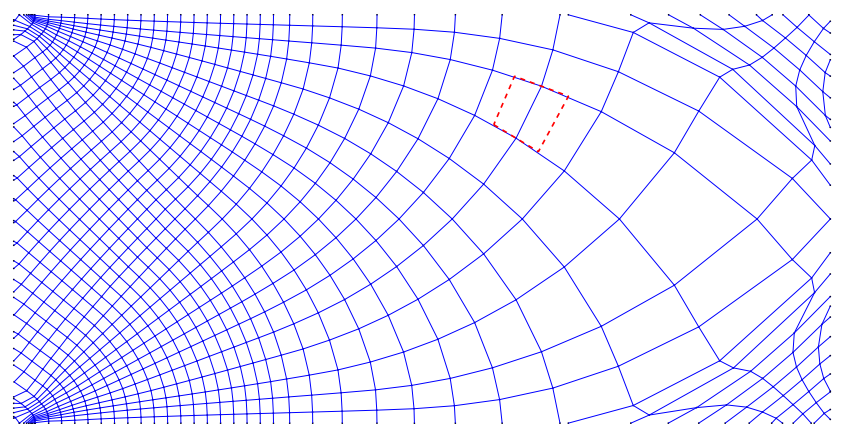

(a) Polygon around element to integrate volume

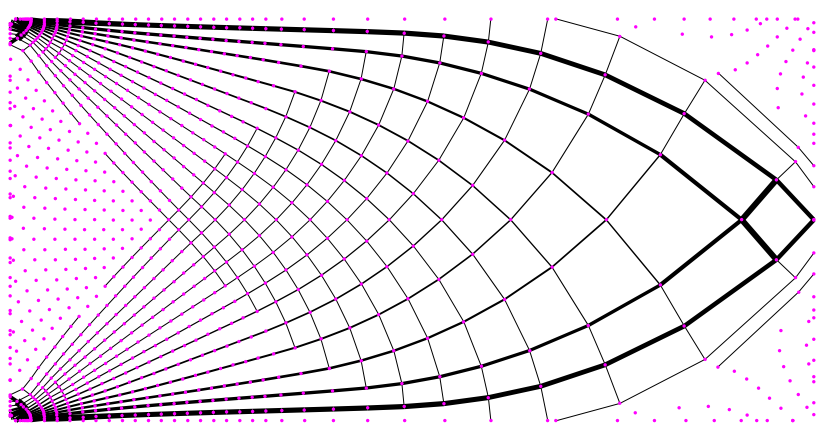

(b) Material mapped to elements

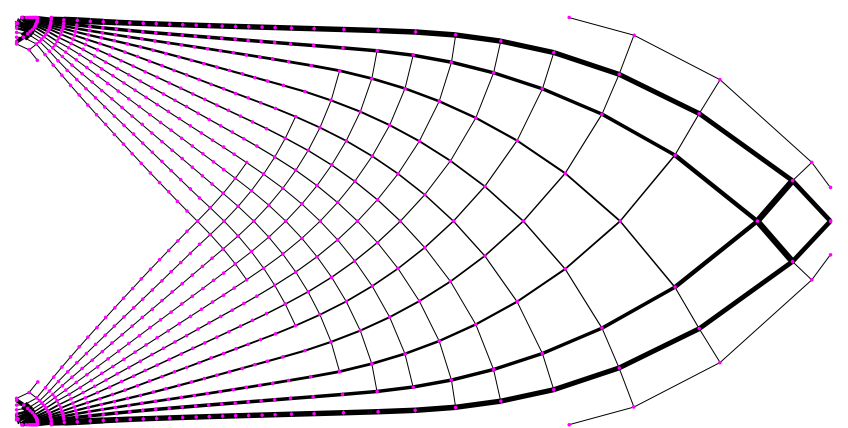

(c) Removal of elements with area smaller than $A_{\eta}$

Fig. 5: Procedure for mapping material from the continuum solution of homogenization-based topology optimization to discrete frame elements

\subsection{Assigning boundary conditions}

A distinction is made between how two different types of boundary conditions (BC's) are applied to the frame structure. Most nodes to which a BC needs to be assigned (in this work referred to as standard-BC's) can be identified easily, e.g. by finding the intersection with a line along which the BC's have to be applied. However, the analytical solutions for Michell's problem of least-weight trusses (Hemp, 1973; Lewiński et al., 1994a b; Lewiński and Rozvany, 2008), also consists of boundary conditions that can be interpreted as source points from which multiple elements originate. These so-called fan-BC's can be found for example at the nodal Dirichlet BC's in the analytical solution of the Michell cantilever example (Lewiński et al. 1994b). Careful inspection of the contour lines obtained for this example (Figure 4(b)) reveals that multiple contour lines point to the location where these BC's have to be applied. To allow for these fan-BC's in the initial structure, all elements and nodes within radius $R_{B C}=1 / 8 L$ of both BC's are pulled exactly into the boundary nodes, as is shown in Figure 6 .

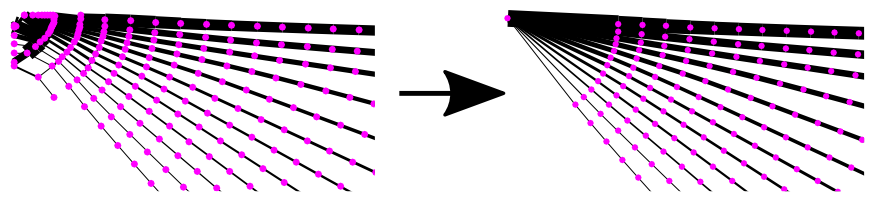

Fig. 6: Operation that pulls in all nodes and elements within $R_{B C}=100 h_{f}$ to generate a fan-BC

We can automatically identify if a boundary condition needs to be a standard-BC or a fan-BC. Therefore, we look at orientation field $\theta$, inside radius $R_{B C}$ of the boundary nodes. At locations where a fan- $B C$ needs to be inserted, the angular field is rapidly changing, hence we can simply check if points close to a nodal BC are inside $\Omega_{\theta}$.

\subsection{Preparation of initial frame structure}

To clean up and improve the stability of the initial structure, normal nodes (i.e. non-boundary conditions) connected to only one or two elements are removed. In the case of a node connected to a single element, the element cannot carry any load, hence both node and element are removed. Furthermore, connections between two elements are unstable and do not exist in the solution space for Michell's problem of least-weight trusses. Therefore the corresponding elements are merged into a 
single element, while the node is removed. In some situations this operation will result in crossing elements. If this happens the largest element in a crossing is removed. Finally, it has to be mentioned that all operations that modify the structure, e.g. assigning BC's, are made volume preserving. Hence, the relative material distribution throughout the domain remains as close to the material distribution of the continuum model as possible.

The initial frame structure for the Michell cantilever obtained using the approach described above, for $\varepsilon=$ $50 h_{f}$ can be seen in Figure 7(a). Similarly, the initial structures for the MBB-beam and the Michell cantilever with circular support, both for $\varepsilon=50 h_{f}$, can be seen in Figures 7(b) and (c) respectively.

\subsection{Void regions inside design domain}

The mapping procedure described above can easily be extended to take specified void regions in the design domain into account. However, special care needs to be taken at the corners of these void regions, since the homogenization based microstructures are either oriented parallel to the void region, or oriented to go exactly through the corner point of a void region. This highly optimal use of the available design space can result in mapped elements that cross the void domain. Furthermore, it should be noted that corners of a void region can also be a source of a fan similar to the fan-BC's. As discussed by Lewiński and Rozvany (2008) the optimal solution for the L-shaped domain, shown in Figure 2(c), consists of a fan at the corner of the void domain.

To accommodate for these fan corner nodes, a check is performed for each corner of a specified void domain. If points within $R_{B C}$ of these corners are inside $\Omega_{\theta}$, a fan is created in the exact same manner as a fan-BC. At a corner of the void domain, which is not a fan, and at which an element crosses, a node is inserted and the crossing element is split in two elements.

The projected frame structure for the L-shaped domain can be seen in Figure 8 (a), the initial frame structure for the MBB-beam with a rectangular region of void is shown in Figure 8(b), where for both structures $\varepsilon=50 h_{f}$.

\section{Post size- and geometry-optimization}

In this section we propose a frame optimization scheme that ensures solutions close to the solutions of Michell's problems of least-weight trusses, hence with a negligible bending contribution. Furthermore, we propose a

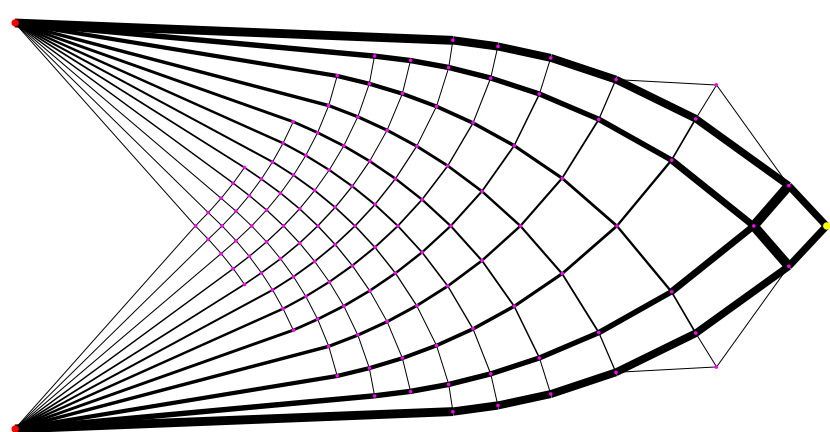

(a) Michell cantilever

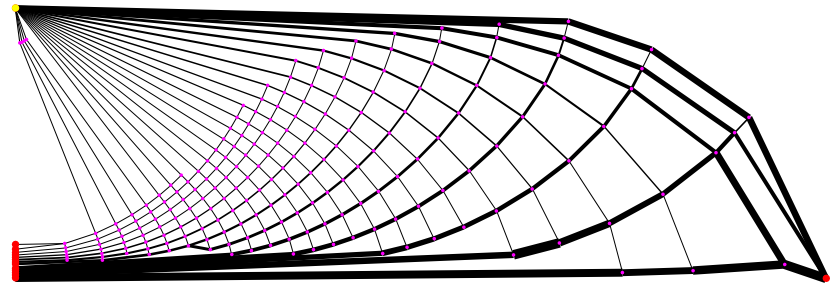

(b) MBB-beam

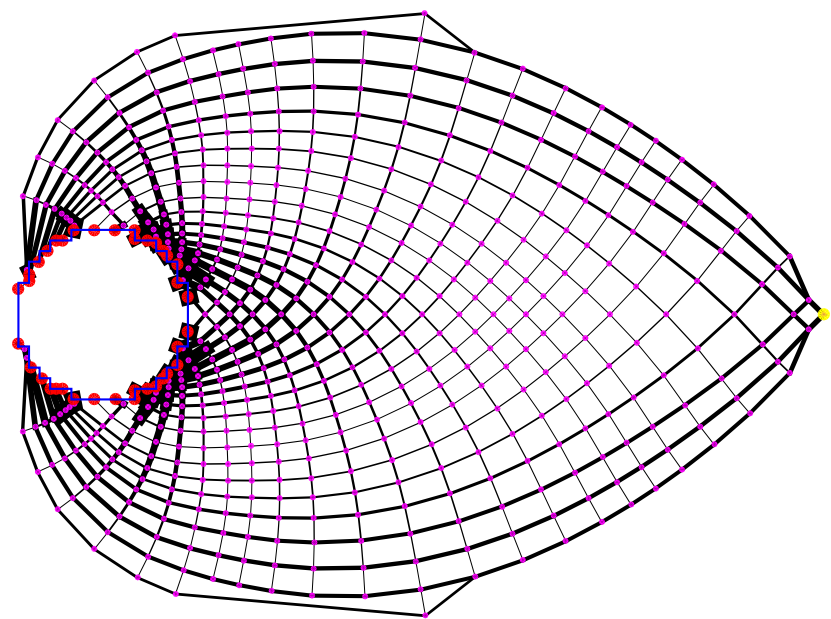

(c) Michell cantilever with circular support

Fig. 7: Initial structures extracted from homogenization-based topology optimization for $\varepsilon=50 h_{f}$

strategy to avoid: 1) elements that are very thin, 2) elements that are very short, and 3) elements that are parallel and partially overlap, since each of these three cases result in a singular stiffness matrix when modeled as truss.

\subsection{Motivation for frame analysis}

It is well-known that solutions of Michell's problem of least-weight trusses are in the space of statically determinate structures, (Pedersen, 1969). However, it is 


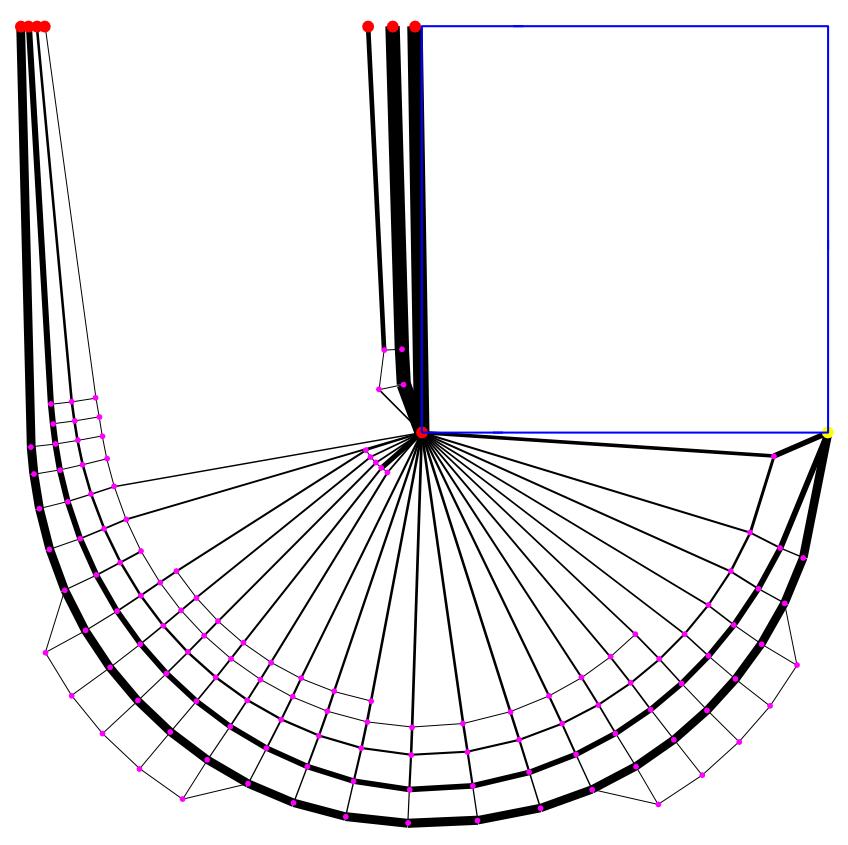

(a) L-shaped domain

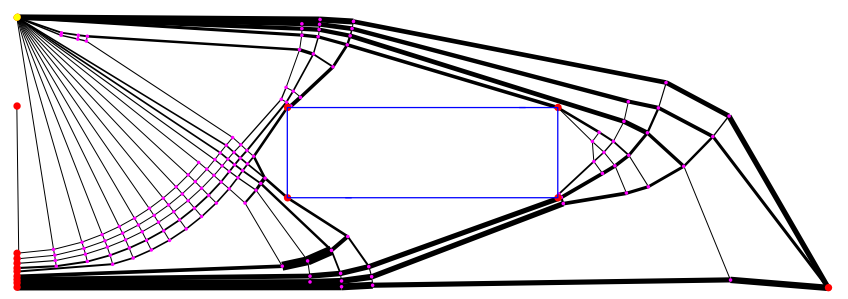

(b) MBB-beam with a rectangular region of void

Fig. 8: Initial structures extracted from homogenization-based topology optimization for $\varepsilon=50 h_{f}$

not possible to use truss elements to assess the performance of the mapped structures. Small misalignments close to boundary conditions, e.g. at the symmetry conditions for the MBB-beam example, may result in indeterminate structures. Nevertheless, a post-optimization scheme is required, such that the projected structures converges towards solutions of Michell's problem of leastweight trusses. To do so, frame elements are used that, contrary to truss elements, not only carry axial loads but also have bending stiffness.

For such an analysis appropriate relations between the domain length $L$ and element areas have to be chosen, such that the bending stiffness does not become dominant. A circular cross-section is chosen for the frame elements, hence for given element $i$ the relation between axial $\left(k_{a}\right)$ and bending $\left(k_{b}\right)$ stiffness is given by,

$\frac{k_{a}}{k_{b}} \propto \frac{l_{i}^{2}}{A_{i}}$ where $l_{i}$ is the length of the element and $A_{i}$ the corresponding area.

The design vector for geometry optimization $\mathbf{x}_{n}$ holds the coordinates of the nodal positions. To get a well conditioned optimization problem the length of the domain is scaled such that $\mathbf{x}_{n} \in[0,10]$. The vector of element areas $\mathbf{A}$ is obtained using the design vector for size optimization $\mathbf{x}_{e}$, and the maximum allowable element area $A_{\max }$.

$\mathbf{A}\left(\mathbf{x}_{e}\right)=\mathbf{x}_{e} A_{\max } \quad \forall \mathbf{x}_{e} \in[0,1[$

Here $\mathbf{x}_{e}$ is based on the relative material distribution in the mapped elements. The relative values in $\mathbf{x}_{e}$ are scaled down far enough such that the upper bound of 1 never becomes active to prevent that $\mathbf{A}>A_{\max }$. Therefore, the relation between bending and axial stiffness is only controlled by choosing an appropriate value for $A_{\max }$. Hence, $A_{\max }$ is chosen differently for each optimization example such that the relation between the mean values of $\frac{k_{a}}{k_{b}}\left(\right.$ i.e. $\left.\frac{\overline{k_{a}}}{k_{b}}\right)$ is always exactly the same at the start of the post-optimization scheme, e.g. $\frac{\overline{k_{a}}}{k_{b}}=100$.

To converge towards a design that is purely loaded in axial direction, the relative importance of the bending stiffness is slowly decreased using a continuation scheme. In this scheme $A_{\max }$ and the volume constraint $V^{*}$ are lowered by $12.5 \%$ for every 10 iterations. This does not have an effect on the relative distribution of axial loads; however, it does make it uneconomical to have elements that are not purely loaded in axial direction. The continuation scheme start after the first 100 iterations and is continued until $A_{\max }$ is less than $2.5 \%$ of its initial value. At this point the contribution of the bending stiffness to the strain energy is negligible, as will be discussed in more detail in Section 5. The choice for the steps used in the continuation scheme result from a trade-off between the performance of the design and computational cost. For smaller steps, significantly more iterations are required to optimize the design, resulting in a slightly better objective. Similarly, a larger stepwise reduction in bending stiffness, means that the algorithm converges more quickly; however, resulting in a reduced performance.

\subsection{Optimization scheme}

The frame optimization problem is solved to minimize compliance $\mathcal{J}_{f}$, subject to a volume constraint $V^{*}$, which is the amount of material in the frame members at the first iteration. The optimization problem is solved in nested form using a gradient-based optimization scheme, where we use the Method of Moving Asymptotes (MMA) 
to update the design variables (Svanberg, 1987). The corresponding optimization problem can be written as,

$$
\begin{aligned}
\min _{\mathbf{x}_{n}, \mathbf{x}_{e}} & : \mathcal{J}_{f}\left(\mathbf{x}_{n}, \mathbf{x}_{e}, \mathbf{U}\right)=\mathbf{F}^{T} \mathbf{U} \\
\text { s.t. } & : \mathbf{K}\left(\mathbf{x}_{n}, \mathbf{x}_{e}\right) \mathbf{U}=\mathbf{F} \\
& : \frac{\sum_{i=1}^{n_{e}} l_{i}\left(\mathbf{x}_{n}\right) A_{i}\left(\mathbf{x}_{e}\right)}{V^{*}}-1 \leq 0 \\
& : \mathbf{x}_{n, l} \leq \mathbf{x}_{n} \leq \mathbf{x}_{n, u} \\
& : \mathbf{x}_{e, l} \leq \mathbf{x}_{e} \leq \mathbf{x}_{e, u}
\end{aligned}
$$

Where $\mathbf{x}_{e, l}, \mathbf{x}_{e, u}, \mathbf{x}_{n, l}$ and $\mathbf{x}_{n, u}$ are the lower and upper bounds for the size and geometry design vectors respectively. For each design iteration lower and upper bounds on the design variables are adaptively selected. These bounds are chosen such that the design changes gradually, furthermore, the bounds on $\mathbf{x}_{n}$ make sure that elements will not cross each other, or move into the specified void domains.

\subsection{Removal of thin elements}

During the optimization, values in $\mathbf{x}_{e}$ can become close to 0 , hence these elements contain almost no material. Typically, these are prevented by using a lower bound on the areas; however, this adds artificial stiffness to the structures, and can also prevent structures from becoming statically determinate. Therefore, elements smaller than a selected threshold will be removed from the solution space. This threshold is based on the value of $A_{\eta}$ used in the mapping procedure, but scaled with the same factor used to obtain $\mathbf{x}_{e}$. Furthermore, the threshold is consistently updated during the continuation scheme.

By removing thin elements from the frame mesh, normal nodes (i.e non-BC's) can be connected to only one or two elements, making the structure unstable. To avoid this undesired effect, the exact same procedure is applied as discussed in Section 3.5 .

\subsection{Merging of close nodes}

It is well-known that for some elements the nodes move towards the same point, making the corresponding element length zero. This effect, sometimes referred to as melting nodes (Achtziger, 2007), will cause a singularity in the stiffness matrix and is therefore undesired. To take this into account, the nodes of elements shorter than a selected threshold $l_{\text {short }}$ will be merged, removing a node from the solution space in an approach similar to (He and Gilbert, 2015). The value for the threshold $l_{\text {short }}$ is selected to be one fifth of the average size of the projected element $\varepsilon$, scaled with the same scaling factor used to obtain $\mathbf{x}_{n}$.

By merging nodes, it is possible that non-unique elements exist between two nodes. To take this undesired effect into account, one of the corresponding elements is removed, and the volume of both elements is contained by the remaining element.

4.5 Merging of parallel and partially overlapping elements

It is possible that during post-optimization two or more elements, located along boundaries of the design domain are partially overlapping and parallel. This situation can be observed at the lower boundary of the MBB-beam example with a void, shown in Figure 9 Here, there is an element between node 1 and node 2, an element between node 2 and node 3 , and an element between node 1 and node 3 .

Although all three elements are unique, it is unphysical that the elements overlap, furthermore, this can have an undesired effect on the condition-number of the stiffness matrix. To remedy this, the longest element is split into two elements. In this case the two smaller elements already exist, and the volume of the longest elements is transferred to the smaller elements consistently.

\section{Numerical examples}

An overview of all parameters used in this work can be found in Table 1. The horizontal lines are used to show a division in parameters used in: 1) homogenizationbased topology optimization, 2) calculation of mapping functions, 3) post-optimization. Please note that we use $\varepsilon=50 h_{f}$, unless otherwise stated. In the following, we demonstrate the suggested procedure on a number of examples and compare with analytical solutions when available.

\subsection{Michell cantilever}

The near-optimal initial structure for the Michell cantilever, shown in Figure 7(a), has been optimized using the presented post-optimization scheme. The result, shown in Figure 10 can be modeled as a truss, i.e. it is statically determinate.

To assess the performance of the optimized design, one can look at the non-dimensional mass when evaluated as truss $\bar{M}_{t}$, which for a Michell cantilever can be 


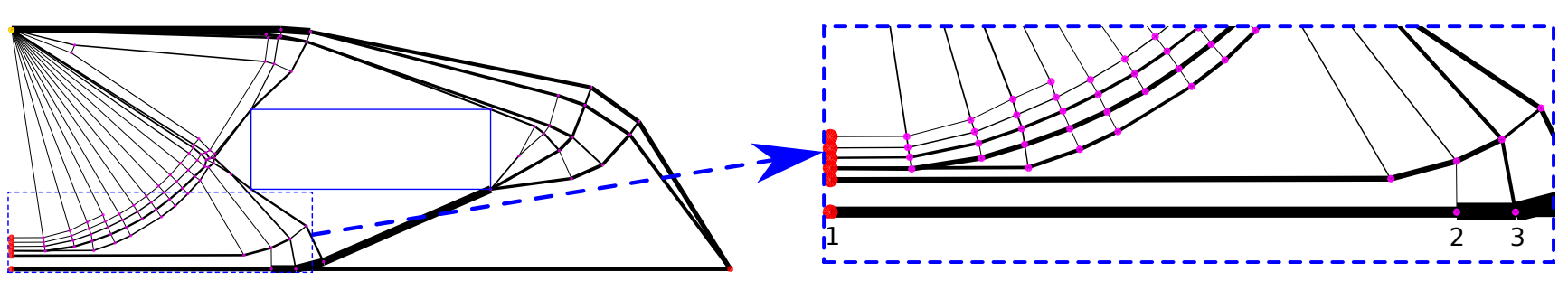

Fig. 9: MBB-beam with a rectangular region of void, to demonstrate that elements can be parallel and partially overlap. There is an element between node 1 and node 2 , an element between node 2 and node 3 , and an element between node 1 and node 3

Table 1: Parameters used in the numerical experiments

\begin{tabular}{lll}
\hline Parameter & Definition & Value \\
\hline$E$ & Young's modulus of material in continuum model & 1 \\
$E_{\text {min }}$ & Young's modulus of background material & $0.005 E$ \\
$\nu$ & Poisson's ratio for isotropic background material & 0.3 \\
$r_{\text {min }}$ & Filter radius used in continuum topology optimization & $1.5 h_{c}$ \\
$\eta$ & Minimum feature size per layer in the microstructure & 0.05 \\
\hline$\gamma_{\theta}$ & Threshold value that determines whether angular field is rapidly changing & $\frac{\pi}{4} \frac{1}{h_{c}}$ \\
$\alpha_{1}$ & Spatially variant parameter to relax the objective of projection & {$[0.01,0.1,1]$} \\
$\alpha_{2}$ & Spatially variant parameter to relax the constraint of projection & {$[0,1]$} \\
$\varepsilon$ & average length of the frame member & $50 h_{f}$ \\
$R_{B C}$ & Radius to search for a fan- $B C$, and to pull in all nodes to BC & $1 / 8 L$ \\
\hline$l_{\text {short }}$ & Short element length which dictates when two nodes are merged & $0.2 \varepsilon$ \\
$\frac{k_{a}}{k_{b}}$ & Starting relation between the mean axial and bending stiffness & 100 \\
$\frac{A_{\text {max }, \text { nd }}}{A_{\text {max start }}}$ & Measure for reduction in bending stiffness & 0.025 \\
\hline
\end{tabular}

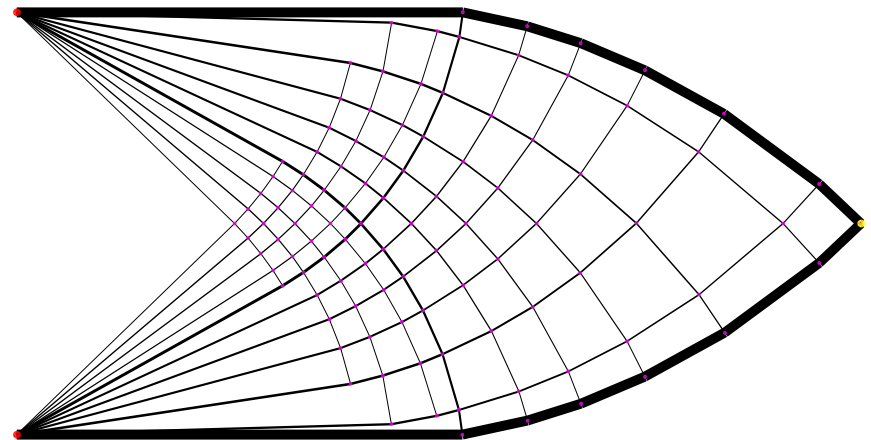

Fig. 10: Optimized structure for the Michell cantilever, using initial structure for $\varepsilon=50 h_{f}$

calculated as (Rozvany, 1998; Bendsøe et al., 1994),

$\bar{M}_{t}=\frac{\sqrt{M E \mathcal{J}_{t}}}{F L}$

Here $M$ is the volume of the final structure, and $\mathcal{J}_{t}$ the compliance when modeled as a truss structure. The non-dimensional mass for the optimized Michell cantilever is 7.0391, which is close to the optimal value, $\bar{M}_{o p t}=7.0247$, found in a table in Graczykowski and Lewiński (2010). This means that the non-dimensional mass of the optimized structure is just $0.204 \%$ higher than the analytical optimum. Furthermore, the compli- ance when the structure is modeled as a truss $\mathcal{J}_{t}$, is almost identical to the compliance modeled as a frame structure $\mathcal{J}_{f}=7.0390$, used in the post-optimization scheme. It is possible to identify a measure $\left(f_{b}\right)$ of the total contribution of the bending stiffness on $\mathcal{J}_{f}$.

$f_{b}=\frac{\mathcal{J}_{f}-\mathbf{U}_{t}^{T} \mathbf{F}_{t}}{\mathcal{J}_{f}} \times 100 \%$

Here $\mathbf{U}_{t}$ and $\mathbf{F}_{t}$ correspond to the displacement indices of the solution and load vector respectively, i.e. excluding indices corresponding to rotation. The bending contribution for the optimized Michell cantilever is $0.00045 \%$. A plot of this contribution for each iteration of the post-optimization scheme can be seen in Figure 11.

Due to the continuation scheme, the bending contribution is lowered every 10 iterations after the $100^{t h}$ iteration. However, it is more interesting to see that the bending contribution is drastically reduced in the first few iterations. The reason is that tiny misalignments of nodal positions close to the boundary conditions severely deteriorate the performance of the structure (e.g. singular matrix when modeled as truss). When modeled as a frame structure, the initial bending stiffness provides stability; however, the performance is improved when these nodes are better aligned, hence the 


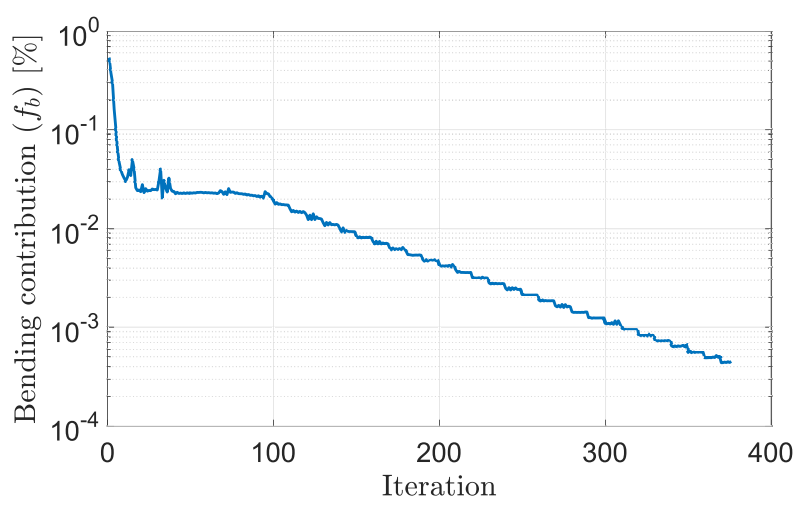

Fig. 11: Contribution of the bending stiffness to the overall compliance for the Michell cantilever

contribution of the bending to the compliance is reduced.

The optimization is performed for the Michell cantilever using three different levels of detail of the initial structure, i.e. $\varepsilon=20 h_{f}, \varepsilon=50 h_{f}$, and $\varepsilon=100 h_{f}$. The optimized structures for $\varepsilon=20 h_{f}$, and $\varepsilon=100 h_{f}$ can be seen in Figure 12 (a) and (b) respectively.

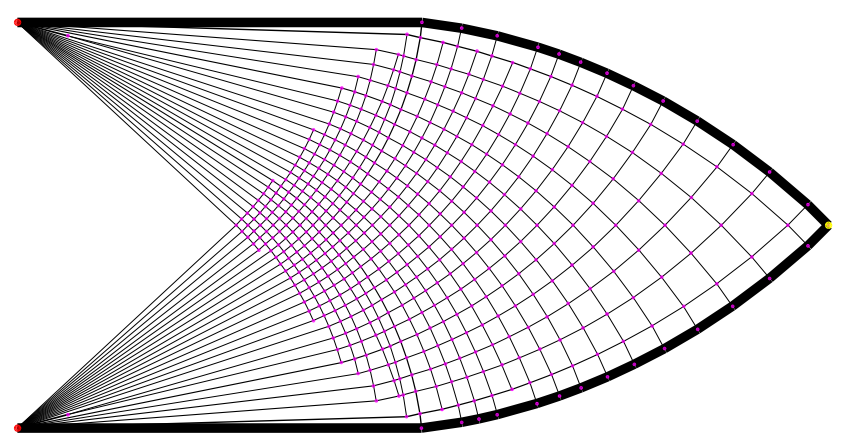

(a) $\varepsilon=20 h_{f}$

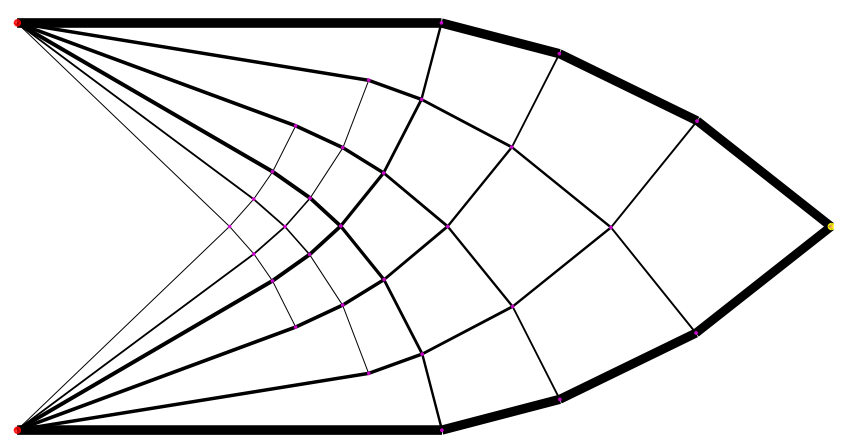

(b) $\varepsilon=100 h_{f}$

Fig. 12: Optimized structures for the Michell cantilever

The corresponding size of the fine mesh on which the nodes and elements are obtained, the number of nodes
$N_{n}$, number of elements $N_{e}$, the performance measured in non-dimensional masses $\left(\bar{M}_{f}\right.$ and $\left.\bar{M}_{\text {opt }}\right)$, the error $\xi$ between $\bar{M}_{f}$ and the analytical solution $\bar{M}_{o p t}$ and the contribution of the bending stiffness $f_{b}$ can be found in Table 2. As expected, a finer initial structure results in a better performing design. Furthermore, the time to do the homogenization-based topology optimization on the coarse mesh $T_{c}$, the time to obtain the initial structure $T_{\phi}$, the time to do the post-optimization $T_{f}$, and the total time $T_{t o t}$ are shown. Here it has to be noted that all experiments are performed using a single processor MATLAB code on a standard PC. Hence, large potential for further time reduction exists.

It can be seen that a more detailed structure comes at a larger computational cost, which is dominated by the sensitivity analysis for the post-optimization scheme. Furthermore, the increased level of detail means an increase in computational cost to obtain the initial mesh, since more contour lines of mapping function $\phi_{1}$ and $\phi_{2}$ need to be considered. Nevertheless, the increase in computational cost is significantly smaller compared to growth methods, where the time to insert a new member scales exponentially.

\subsection{Stability of optimized results}

The far majority of the optimized structures for the other examples is unstable when modeled as a truss. To explain this we can take another look at Figure 9, where the bottom of the MBB-beam with a rectangular void is shown. In this example, a condition for stability when modeled as truss, is that node 1 and node 2 , should have the exact same value for their $y$-coordinate. Even the slightest misalignment (e.g. $10^{-6}$ ) will result in an unstable structure since node 1 , is only supported in the $x$-direction.

When modeled as frame, even the smallest bending contribution, will prevent such an instability. Hence, for the post-optimized MBB-beam shown in Figure 13 . the element that is connected to the middle node of the symmetry boundary is nearly horizontal; however not exactly. Unfortunately, this statical indeterminacy means that we cannot assess the performance as a truss, and hence an exact comparison between the optimal value for the non-dimensional mass, $\bar{M}_{\text {opt }}=14.0937$ and $\bar{M}_{t}$ is not possible. Nevertheless, we argue that the bending contribution is sufficiently small, $f_{b}=0.0055 \%$ to allow for a comparison between $\bar{M}_{\text {opt }}$ and the nondimensional weight calculated with the compliance from the frame model, $\bar{M}_{f}=14.1878$.

To demonstrate this we decrease $A_{\max }$ by different orders of magnitude, such that the bending stiffness of the node at the symmetry boundary is reduced. As can 
Table 2: Performance and computational cost of the near optimal truss and frame structures. Where example 1) is the Michell cantilever, 2) the Michell cantilever with circular support, 3) the MBB-beam, 4) the MBB-beam with void, and 5) the L-shaped domain

\begin{tabular}{|c|c|c|c|c|c|c|c|c|c|c|c|c|}
\hline Ex. & Proj. mesh & $\varepsilon$ & $N_{n}$ & $N_{e}$ & $\bar{M}_{f}$ & $\bar{M}_{o p t}$ & $\xi$ & $f_{b}$ & $T_{c}$ & $T_{\phi}$ & $T_{f}$ & $T_{t o t}$ \\
\hline 1 & $1600 \times 800$ & $20 h_{f}$ & 453 & 900 & 7.0327 & 7.0247 & $0.114 \%$ & $0.00032 \%$ & $96.0 \mathrm{~s}$ & $104.2 \mathrm{~s}$ & $339.9 \mathrm{~s}$ & $540.0 \mathrm{~s}$ \\
\hline 1 & $1600 \times 800$ & $50 h_{f}$ & 103 & 202 & 7.0392 & 7.0247 & $0.206 \%$ & $0.00045 \%$ & $96.0 \mathrm{~s}$ & $22.9 \mathrm{~s}$ & $67.5 \mathrm{~s}$ & $186.4 \mathrm{~s}$ \\
\hline 1 & $1600 \times 800$ & $100 h_{f}$ & 32 & 60 & 7.0545 & 7.0247 & $0.422 \%$ & $0.00059 \%$ & $96.0 \mathrm{~s}$ & $18.4 \mathrm{~s}$ & $29.3 \mathrm{~s}$ & $143.7 \mathrm{~s}$ \\
\hline 2 & $1600 \times 1200$ & $50 h_{f}$ & 380 & 710 & 2.1248 & 2.1401 & $-0.720 \%$ & $0.0173 \%$ & $136.3 \mathrm{~s}$ & $47.0 \mathrm{~s}$ & $288.4 \mathrm{~s}$ & $487.5 \mathrm{~s}$ \\
\hline 2 & $1600 \times 1200$ & $100 h_{f}$ & 122 & 212 & 2.1474 & 2.1401 & $0.340 \%$ & $0.0021 \%$ & $136.3 \mathrm{~s}$ & $30.5 \mathrm{~s}$ & $72.7 \mathrm{~s}$ & $255.3 \mathrm{~s}$ \\
\hline 3 & $2520 \times 840$ & $50 h_{f}$ & 106 & 208 & 14.1878 & 14.0937 & $0.663 \%$ & $0.0055 \%$ & $152.1 \mathrm{~s}$ & $45.1 \mathrm{~s}$ & $94.8 \mathrm{~s}$ & $292.0 \mathrm{~s}$ \\
\hline 3 & $2520 \times 840$ & $100 h_{f}$ & 40 & 74 & 14.2675 & 14.0937 & $1.218 \%$ & $0.0085 \%$ & $152.1 \mathrm{~s}$ & $32.6 \mathrm{~s}$ & $43.8 \mathrm{~s}$ & $228.4 \mathrm{~s}$ \\
\hline 4 & $2520 \times 840$ & $50 h_{f}$ & 40 & 67 & 14.5616 & - & - & $0.0029 \%$ & $146.6 \mathrm{~s}$ & $48.5 \mathrm{~s}$ & $65.8 \mathrm{~s}$ & $260.9 \mathrm{~s}$ \\
\hline 4 & $2520 \times 840$ & $100 h_{f}$ & 19 & 28 & 14.6425 & - & - & $0.0031 \%$ & $146.6 \mathrm{~s}$ & $43.3 \mathrm{~s}$ & $37.8 \mathrm{~s}$ & $227.7 \mathrm{~s}$ \\
\hline 5 & $1600 \times 1600$ & $50 h_{f}$ & 26 & 46 & 9.3004 & 9.283 & $0.187 \%$ & $0.0074 \%$ & $178.4 \mathrm{~s}$ & $53.3 \mathrm{~s}$ & $58.2 \mathrm{~s}$ & $289.8 \mathrm{~s}$ \\
\hline 5 & $1600 \times 1600$ & $100 h_{f}$ & 15 & 24 & 9.3284 & 9.283 & $0.487 \%$ & $0.0044 \%$ & $178.4 \mathrm{~s}$ & $45.8 \mathrm{~s}$ & $37.0 \mathrm{~s}$ & $261.2 \mathrm{~s}$ \\
\hline
\end{tabular}

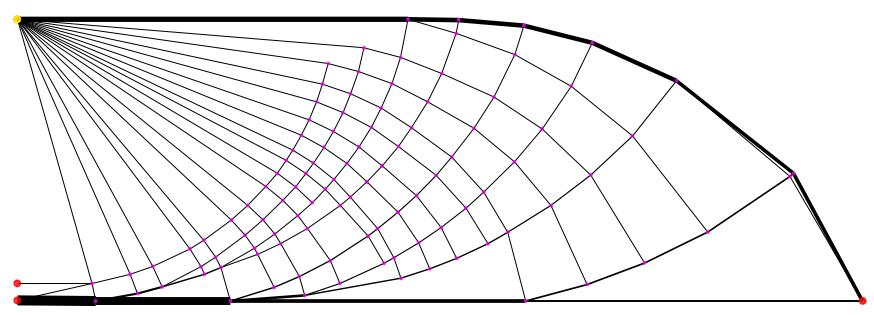

Fig. 13: Optimized structure for the MBB-beam, using initial structure for $\varepsilon=50 h_{f}$

be seen in Table 3 , this will lead to an increase in nondimensional mass of the frame model $\left(\bar{M}_{f}\right)$. However, the contribution of the purely axial stiffness $\bar{M}\left(\mathbf{U}_{t}^{T} \mathbf{F}_{t}\right)$ remains almost perfectly intact. The reason for this is that the node at the symmetry boundary is the only node that causes an instability. Only this node is affected by a decrease in bending stiffness, hence the increase in strain energy in the system is purely due to the near horizontal element being stretched. This increase in energy has a negligible effect on the energy in the rest of the system, even for low values of $A_{\max }$, and therefore we argue that $\bar{M}_{f}$ can be used to compare with the optimal solution $\bar{M}_{o p t}$.

Table 3: Non-dimensional mass of the frame structure $\bar{M}_{f}$, and purely axial contribution of the nondimensional mass $\bar{M}\left(\mathbf{U}_{t}^{T} \mathbf{F}_{t}\right)$ when the area is scaled down

\begin{tabular}{cccc}
\hline Area scaling & $\bar{M}_{f}$ & $\bar{M}\left(\mathbf{U}_{t}^{T} \mathbf{F}_{t}\right)$ & $f_{b}$ \\
\hline$A_{\max }$ & 14.1878 & 14.1870 & $0.0055 \%$ \\
$10^{-1} A_{\max }$ & 14.1890 & 14.1890 & $0.0050 \%$ \\
$10^{-2} A_{\max }$ & 14.1940 & 14.1890 & $0.0340 \%$ \\
$10^{-3} A_{\max }$ & 14.2370 & 14.1890 & $0.3360 \%$ \\
$10^{-4} A_{\max }$ & 14.6600 & 14.1890 & $3.2120 \%$ \\
$10^{-5} A_{\max }$ & 18.3610 & 14.1900 & $22.7140 \%$ \\
$10^{-6} A_{\max }$ & 39.4440 & 14.3250 & $63.6830 \%$ \\
\hline
\end{tabular}

\subsection{Discussion of results}

The post-optimized structures for the MBB-beam, Michell cantilever with circular support, MBB-beam with rectangular void and L-shaped domain, all for $\varepsilon=50 h_{f}$ can be seen in Figure 13 , Figure 14(a), (b) and (c) respectively. While the corresponding performance and the different times can be seen in Table 2. It is interesting to see that the optimized structures perform very close to the optimal solution, at a negligible bending contribution. However, the performance of the MBBbeam with rectangular void cannot be compared to an analytical solution, since this solution is not known.

Furthermore, it is interesting to see that the nondimensional mass for the Michell cantilever with circular support is lower than the analytical optimum. This does not come from the fact that it is modeled as a frame, nor does it come from the analytical solution being wrong. The simple reason is that the boundary of the extracted structure is not perfectly circular as can be seen in Figure 14(a). This jagged boundary has its origin in the coarse-scale homogenization-based topology optimization model, where the circular boundary is approximated by coarse square elements. In our algorithm, this is the boundary that is transferred down from the coarse-scale topology optimization model to the frame model, hence the difference with the analytical optimum.

Besides the fact that the final structures perform well, it has to mentioned that the total procedure comes at a relatively low computational cost, i.e. all examples have been obtained within 10 minutes using a single processor MATLAB code on a standard PC. The homogenization-based topology optimization can be done in a couple of minutes, where the difference in time between the examples comes from different mesh sizes. E.g. the Michell cantilever is optimized on a mesh of $80 \times 40$ elements, while the L-shaped domain is opti- 


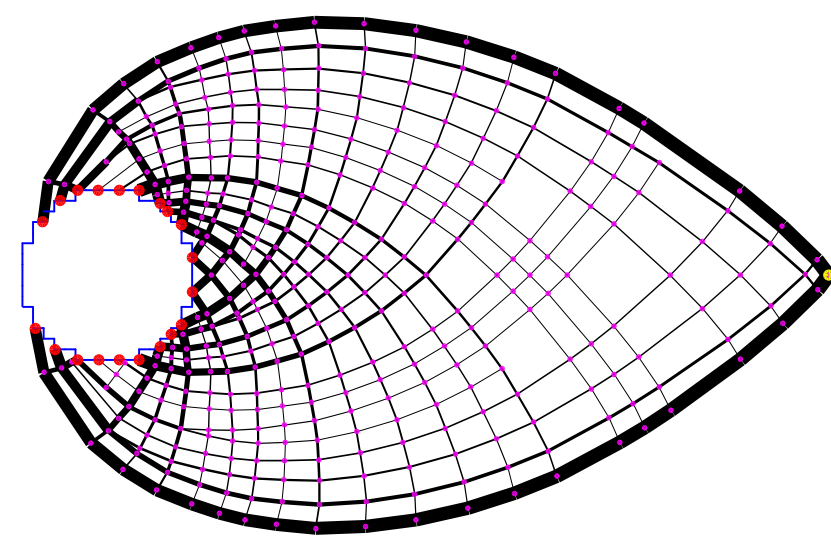

(a) Michell cantilever with circular support

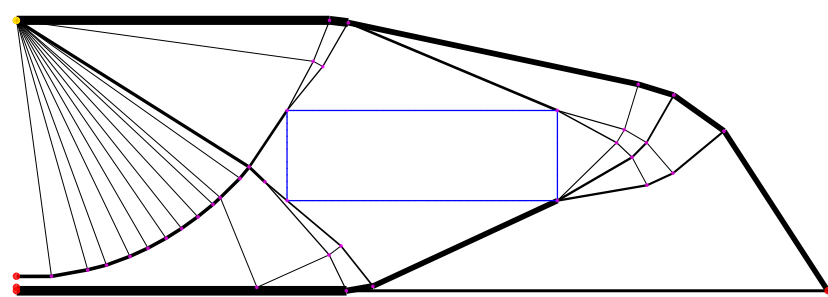

(b) MBB-beam with rectangular void

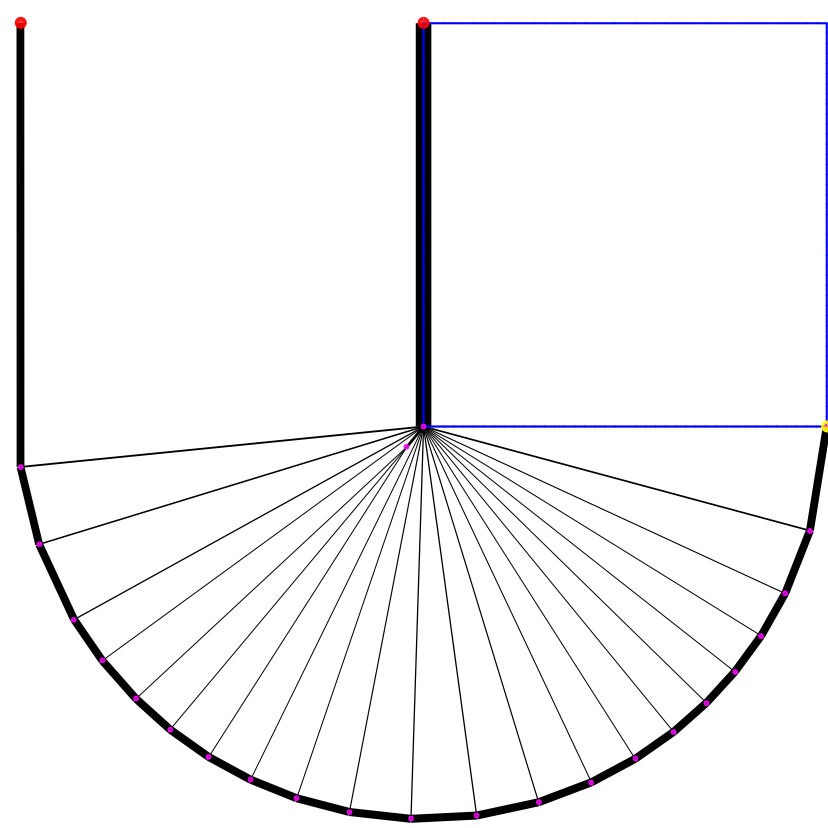

(c) L-shaped domain

Fig. 14: Optimized structures extracted from homogenization-based topology optimization for $\varepsilon=50 h_{f}$

mized on a mesh consisting of $80 \times 80$ elements. The extraction time for the initial structure does increase when the average spacing $\varepsilon$ is decreased. However, this increase in computational cost is more or less quadratically related to $1 / \varepsilon$, which is a significant advantage over growth methods that scale exponentially when fine designs are considered.

\section{Conclusion}

An approach to obtain near-optimal frame structures has been presented, where the discrete structures are based on the solution of a homogenizationbased topology optimization model. The coarse-scale homogenization-based continuum solution is used to create a close to optimal initial structure, which is obtained by solving for two mapping functions and using their corresponding contour lines. Furthermore, accurate integration of the continuum solution allows for a good starting guess for the element areas. Afterwards, these initial structures are optimized using a frame optimization code, to avoid the problem of a singular matrix when modeled as truss. To make sure that the final structures are close to the known solutions of Michell's problem of least-weight trusses, we gradually reduce the bending stiffness such that the final structures are only loaded in axial direction.

Based on numerical experiments, we can conclude that the presented approach produces near-optimal frame structures at a relatively low computational cost. This promising performance paves the way for extending the methodology to multiple load problems. In these problems, the optimal continuum solution is in the space of rank-3 microstructures, compared to the orthogonal microstructures used in the current approach. The extension to 3-dimensions is also possible, based on orthogonal projection of (sub-optimal) truss-like microstructures.

Acknowledgements The authors acknowledge the support of the Villum Fonden through the Villum investigator project InnoTop. The authors would also like to thank Andreas Bærentzen and Niels Aage for valuable discussions during the preparation of the work. Finally, the authors wish to thank Krister Svanberg for providing the MATLAB MMA code.

\section{References}

Aage, N., Nobel-Jørgensen, M., Andreasen, C.S., Sigmund, O., 2013. Interactive topology optimization on hand-held devices. Structural and Multidisciplinary Optimization 47, 1-6. doi $10.1007 /$ s00158-012-0827-z.

Achtziger, W., 2007. On simultaneous optimization of truss geometry and topology. Structural and Multidisciplinary Optimization 33, 285-304. doi 10.1007/ s00158-006-0092-0. 
Bendsøe, M.P., Ben-Tal, A., Zowe, J., 1994. Optimization methods for truss geometry and topology design. Structural optimization 7, 141-159. doi:10. 1007/BF01742459.

Bendsøe, M.P., Haber, R.B., 1993. The michell layout problem as a low volume fraction limit of the perforated plate topology optimization problem: An asymptotic study. Structural optimization 6, 263267. doi 10.1007/BF01743385

Bourdin, B., 2001. Filters in topology optimization. International Journal for Numerical Methods in Engineering 50, 2143-2158. doi $10.1002 / \mathrm{nme} .116$

Bourdin, B., Kohn, R., 2008. Optimization of structural topology in the high-porosity regime. Journal of the Mechanics and Physics of Solids 56, 1043 1064. doi $10.1016 /$ j.jmps.2007.06.002.

Bruns, T., 2006. Zero density lower bounds in topology optimization. Computer Methods in Applied Mechanics and Engineering 196, 566 - 578. doi 10 . 1016/j.cma.2006.06.007.

Bruns, T., Tortorelli, D., 2001. Topology optimization of non-linear elastic structures and compliant mechanisms. Computer Methods in Applied Mechanics and Engineering 190, 3443 - 3459. doi $10.1016 /$ S0045-7825(00) 00278-4

Dobbs, M.W., Felton, L.P., 1969. Optimization of truss geometry. Journal of the Structural Division 95, 2105-2118.

Dorn, W.S., Gomory, R.E., Greenberg, H.J., 1964. Automatic design of optimal structures. Journal de Mecanique 3, 25-52.

Gao, G., yu Liu, Z., bin Li, Y., feng Qiao, Y., 2017. A new method to generate the ground structure in truss topology optimization. Engineering Optimization 49, 235-251. doi $10.1080 / 0305215 X .2016 .1169050$.

Gilbert, M., Tyas, A., 2003. Layout optimization of large-scale pin-jointed frames. Engineering Computations 20, 1044-1064. doi 10.1108/ 02644400310503017 .

Graczykowski, C., Lewiński, T., 2010. Michell cantilevers constructed within a half strip. tabulation of selected benchmark results. Structural and Multidisciplinary Optimization 42, 869-877. doi $10.1007 /$ s00158-010-0525-7.

Groen, J.P., Sigmund, O., 2017. Homogenization-based topology optimization for high-resolution manufacturable micro-structures. International Journal of Numerical Methods in Engineering , 1-18doi 10. 1002/nme.5575.

He, L., Gilbert, M., 2015. Rationalization of trusses generated via layout optimization. Structural and Multidisciplinary Optimization 52, 677-694. doi:10. $1007 / \mathrm{s} 00158-015-1260-\mathrm{x}$
Hemp, W.S., 1973. Optimum structures. Clarendon Press Oxford.

Lewiński, T., Rozvany, G.I.N., 2008. Exact analytical solutions for some popular benchmark problems in topology optimization iii: L-shaped domains. Structural and Multidisciplinary Optimization 35, 165174. doi:10.1007/s00158-007-0157-8.

Lewiński, T., Zhou, M., Rozvany, G., 1994a. Extended exact least-weight truss layouts - part ii: Unsymmetric cantilevers. International Journal of Mechanical Sciences 36, 399 - 419. doi $10.1016 / 0020-7403(94)$ 90044-2.

Lewiński, T., Zhou, M., Rozvany, G., 1994b. Extended exact solutions for least-weight truss layouts - part i: Cantilever with a horizontal axis of symmetry. International Journal of Mechanical Sciences 36, 375 398. doi 10.1016/0020-7403(94)90043-4.

Martínez, P., Martí, P., Querin, O.M., 2007. Growth method for size, topology, and geometry optimization of truss structures. Structural and Multidisciplinary Optimization 33, 13-26. doi:10.1007/ s00158-006-0043-9.

Michell, A., 1904. The limits of economy of material in frame-structures. Philosophical Magazine 8, 589-597. doi: $10.1080 / 14786440409463229$.

Pantz, O., Trabelsi, K., 2008. A post-treatment of the homogenization method for shape optimization. SIAM Journal on Control and Optimization 47, 1380-1398. doi:10.1137/070688900.

Pedersen, P., 1969. On the minimum mass layout of trusses, in: AGARD Conf. Proc. No. 36, Symposium on Structural Optimization, pp. 36-70.

Pedersen, P., 1989. On optimal orientation of orthotropic materials. Structural optimization 1, 101106. doi:10.1007/BF01637666.

Pedersen, P., 1990. Bounds on elastic energy in solids of orthotropic materials. Structural optimization 2, 55-63. doi 10.1007/BF01743521.

Ramos Jr., A.S., Paulino, G.H., 2016. Filtering structures out of ground structures - a discrete filtering tool for structural design optimization. Structural and Multidisciplinary Optimization 54, 95-116. doi:10.1007/s00158-015-1390-1.

Rozvany, G.I.N., 1998. Exact analytical solutions for some popular benchmark problems in topology optimization. Structural optimization 15, 42-48. doi:10. 1007/BF01197436.

Rule, W.K., 1994. Automatic truss design by optimized growth. Journal of Structural Engineering 120, 3063 3070 .

Sokół, T., 2011. A 99 line code for discretized michell truss optimization written in mathematica. Structural and Multidisciplinary Optimization 43, 181- 
190. doi:10.1007/s00158-010-0557-z

Svanberg, K., 1987. The method of moving asymptotes - a new method for structural optimization. International Journal for Numerical Methods in Engineering 24, 359-373. doi 10.1002/nme.1620240207.

Washizawa, T., Asai, A., Yoshikawa, N., 2004. A new approach for solving singular systems in topology optimization using krylov subspace methods. Structural and Multidisciplinary Optimization 28, 330339. doi $10.1007 / \mathrm{s} 00158-004-0439-3$.

Zegard, T., Paulino, G.H., 2014. Grand - ground structure based topology optimization for arbitrary $2 \mathrm{~d}$ domains using matlab. Structural and Multidisciplinary Optimization 50, 861-882. doi $10.1007 /$ s00158-014-1085-z.

Zhou, K., Li, X., 2008. Topology optimization for minimum compliance under multiple loads based on continuous distribution of members. Structural and Multidisciplinary Optimization 37, 49-56. doi 10.1007/ s00158-007-0214-3.

Zhou, K., Li, X., 2011. Topology optimization of trusslike continua with three families of members model under stress constraints. Structural and Multidisciplinary Optimization 43, 487-493. doi $10.1007 /$ s00158-010-0584-9. 